\title{
The benefits of apolipoprotein E $\varepsilon 4$ screening to research
}

\section{Serge Gauthier}

ß See related article page 863

$\mathrm{I}$ $\mathrm{n}$ this issue of $C M A \mathcal{}$, Hsiung and colleagues report results from a subanalysis of the data generated by the Canadian Study of Health and Aging (CSHA). ${ }^{1}$ The CSHA involved more than 10000 Canadians aged 65 and over, and it has given us prevalence and incidence figures for dementia in Canada in the early 1990s. In a few years we will be able to determine from the CSHA data the modulating effects on dementia of higher levels of education in early life; ${ }^{2}$ tighter control of systolic hypertension in mid-life; ${ }^{3}$ greater socialization, ${ }^{2}$ leisure activities and physical activity in late life ${ }^{2}$ and increased consumption of fish, red wine and vegetables containing folic acid. ${ }^{4,5}$ These factors have all been shown to be associated with lower risk for dementia.

But we cannot change our genes, and Hsiung and colleagues have evaluated the impact of the apolipoprotein $\mathrm{E}$ $\varepsilon 4$ (ApoE ع4) mutation on the risk of converting from normal to a state of "cognitive impairment no dementia" (CIND) and from CIND to Alzheimer's disease. They show that the presence of this mutation is not associated with an increased risk for CIND. However, subjects who are already cognitively impaired and who carry the mutation are at higher risk for subsequent Alzheimer's disease.

The study by Hsiung and associates is the first large population-based study to reproduce findings that were first noted in highly selected patients who had amnestic "mild cognitive impairment" (MCI) and in whom the presence of 1 or 2 copies of the ApoE $\varepsilon 4$ mutation was associated with an increased risk for subsequent Alzheimer's disease. This is the reason why randomized clinical trials aimed at delaying conversion from amnestic MCI to Alzheimer's disease should distribute patients carrying the ApoE $\varepsilon 4$ mutation equally in the different treatment arms. One of the main difficulties for researchers evaluating interventions that might prevent or delay the onset of dementia is the long time frame needed for follow-up. The study by Hsiung and colleagues points to another option. By enrolling older patients with CIND who carry the ApoE $\varepsilon 4$ mutation and who can be expected to convert to Alzheimer's disease, the trial length could be shortened and useful interventions detected earlier, over a relatively short time frame ( 2 or 3 years). Although conclusions from such a trial would be limited to that segment of the population and not applicable to the population as a whole, it could allow for "proof of principle" of a disease-modifying therapy in high-risk populations.

Is there a clinical role now for ApoE $\varepsilon 4$ testing? Until we have effective and safe prevention and treatment strategies for Alzheimer's disease, it would be inappropriate to use ApoE $\varepsilon 4$ genotype testing in clinical practice. When patients, especially those with a family history of early-onset dementia or Alzheimer's disease, enquire about the role of genetics, it would be useful to remind them of other strategies that might be helpful and will certainly do no harm. These strategies include reducing systolic blood pressure, increasing physical and social activity, and adopting a diet rich in vegetables containing folic acid (such as green leafy vegetables, oranges, legumes, nuts and whole grain breads), with a regular consumption of fish and a modest intake of red wine. Only when effective prevention and treatment strategies become available will it be appropriate to include ApoE $\varepsilon 4$ - and other mutations that will undoubtedly be identified in the future - in systematic screening for people at increased risk for Alzheimer's disease.

Serge Gauthier is the Director of the Alzheimer's Disease Research Unit, McGill Centre for Studies in Aging, Verdun, Que.

Competing interests: None declared.

\section{References}

1. Hsiung GYR, Sadovnick AD, Feldman H. Apolipoprotein E $\varepsilon 4$ genotype as a risk factor for cognitive decline and dementia: data from the Canadian Study of Health and Aging. CMA7 2004;171(8):863-7.

2. Scarmeas N, Stern Y. Cognitive reserve: implications for diagnosis and prevention of Alzheimer's disease. Curr Neurol Neurosci Rep 2004;4(5):374-80.

3. Skoog I, Gustafson D. Hypertension, hypertension-clustering factors and Alzheimer's disease. Neurol Res 2003;25(6):675-80.

4. Luchsinger JA, Tang MX, Siddiqui M, Shea S, Mayeux R. Alcohol intake and risk of dementia. 7 Am Geriatr Soc 2004;52(4):540-6.

5. Quadri P, Fragiacomo C, Pezzati R, Zanda E, Forloni G, Tettamanti M, et al. Homocysteine, folate, and vitamin B-12 in mild cognitive impairment, Alzheimer disease, and vascular dementia. Am 7 Clin Nutr 2004;80(1):114-22.

Correspondence to: Dr. Serge Gauthier, Director, Alzheimer's Disease Research Unit, McGill Centre for Studies in Aging, Verdun QC H4H 1R2; fax 514 937-9875; serge.gauthier@staff.mcgill.ca 